losen Nadeln. Man reinigt ihn durch Auflösen in Alkohol und Zusetzen von Wasser, bis die Flüssigkeit sich zu trüben beginnt; der neue Harnstoff scheidet sich dann in schönen glänzenden Nadeln aus. Er ist sehr flüchtig. In einer Röhre erhitzt sublimirt er, ohne zu schmelzen, zu einem Netzwerk sehr feiner und leichter Nadeln. Er ist fast unlöslich in Wasser. Er löst sich in Salpetersäure, und diese Lōsung wird durch Wasser gefällt. Er wird durch Kali bei der Temperatur des siedenden Oeles nicht angegrifen.

Lärst man das cyansaure Amylen mit Wasser zusammen stehen, so spaltet es sich zu Kohlensäure und einem Harnstoff, welcher die Zusammensetzung des vorhergehenden besitzt.

Untersuchungen über Isomerie in der Benzoëreihe.

Sechste Abhandlung.

Ueber das Verhalten der Homologen des Benzols gegen Chlor; von F. Beilstein und P. Geitner.

Cannizzaro*) hat bekanntlich die interessante Beobachtung gemacht, dafs das einfach-gechlorte Toluol identisch ist mit der Chlorverbindung des Benzylalkohols. Aus Chlortoluol liefs sich essigsaures Benzyl und Benzylalkohol gewinnen. Abweichend vom indifferenten Chlorbenzol $\mathrm{C}_{6} \mathrm{H}_{5} \mathrm{Cl}$

*) Diese Annalen XCVI, 246. 
kann im Chlortoluol das Chlor leicht in doppelten Zersetzungen ausgewechselt werden. Von der Richtigkeit dieser Angaben hatten wir uns häufig überzeugt. Cannizzaro stellte das Chlortoluol dar durch mehrmaliges Destilliren von Toluol im Chlorstrome. Später schlug man meist den weniger umständlichen Weg ein, und leitete bei gewöhnlicher Temperatur Chlor in Toluol und erhielt ebenfalls das Cannizzaro'sche Chlortoluol. Auch dieses wurde bestätigt, wie denn überhaupt der Eine von uns*) gefunden zu haben glaubte, dafs Chlor auf dieselbe Weise, sowohl in der Kälte als in der Hitze, auf Toluol einwirke. Auffallenderweise blieb aber bei neueren Versuchen die Bildung des Chlortoluols aus. Herr W. Dammann hatte bereits vor $1 \frac{1}{2}$ Jahren im hiesigen Laboratorium ein Präparat erhalten, das keineswegs die von Cannizzaro angegebenen Eigenschaften besafs. Es zeichnete sich vor diesem namentlich durch seine grofse Indifferenz gegen Cyankalium u. s. w. aus. Ein Paar Versuche lieferten uns rasch den Schlüssel zu diesen Erscheinungen. Wir machten nämlich die auffallende Entdeckung, dafs Chlor auf Toluol ganz verschieden einwirkt, je nachdem man es in der Hitze oder in der Kälte darauf einwirken lä/st. Nur im ersteren Fall bildet sich das Cannizzaro'sche Chlortoluol von den bekannten Eigenschaften. Arbeitet man aber in der Kälte, so erhält man ein Chlortoluol, in welchem das Chlor mit derselben Festigkeit gebunden ist, wie im gechlorten Benzol oder in der Chlorbenzoësäure. Bei der Einwirkung des Chlors auf Toluol wird viel Wärme frei. Trägt man daher nicht für besondere Abkühlung Sorge, so kann, namentlich bej Versuchen in gröfserem Mafsstabe, das Toluol sich fast bis zum Sieden erhitzen **). In diesem

*) Diese Annalen CXV1, 338.

**) Aus diesem Verhalten erklärt sich nun sehr natürlich, wie man 
Fall entsteht nur Cannizzaro's Chlortoluol, welches wir, um Verwechselung zu vermeiden, fortan als Chlorbenzyl bezeichnen wollen. Man sieht nun leicht ein, dafs unter gewöhnlichen Verhältnissen sich bei der Einwirkung des Chlors auf Toluol Gemenge von Chlortoluol und Chlorbenzyl in wechselnder Menge bilden werden. Es wird ganz von der Temperatur des Toluols, Menge der Substanz und Geschwindigkeit des Chlorstroms abhängen, ob sich vorzugsweise die eine oder andere Substanz bildet. Wir haben bei unseren ersten Versuchen das Toluol nur durch kaltes Wasser abgekühlt, und erhielten daher stets Chlortoluol, gemengt mit Chlorbenzyl. Man kann sich von der Natur dieses Gemenges sehr leicht durch Behandeln desselben mit Chromsäure überzeugen. Chlorbenzyl geht nämlich dabei in Benzoësäure, das Chlortoluol aber in Chlordracylsäure über, zwei Säuren, die sich durch ihre aufserordentlich verschiedene Löslichkeit in Wasser leicht trennen lassen. Obgleich wir keinen besonderen Versuch angestellt haben, so zweifeln wir doch nicht, dafs wenn das Toluol durch ein Kältegemisch abgekühlt wird, sich nur Chlortoluol bilden wird. Wir haben diesen umständlichen Weg nicht eingeschlagen, weil wir sehr bald ein viel einfacheres Mittel fanden, Chlortoluol chemisch rein darzustellen. Es heruht dieses auf der Anwendung des Jods. Hug o Mülle ${ }^{*}$ ) hat bekanntlich die werthvolle Entdeckung gemacht, dafs die Chlorsubstitutionen bei Gegenwart von Jod viel glatter und regelmäfsiger erfolgen, als durch Chlor allein. Wendet man dieses Verfahren auf Toluol an, leitet man also in mil Jod versetztes Toluol Chlor ein, so beobachtet man

früher (siehe oben) zur Annahme verleitet werden konnte, dafs dss Chlor in der Hitze und in der Kälte gleicbförmig auf Toluol einwirke.

*) Jahresber. für Cbemie u. s. w. f. 1862 , S. 415. 
die interessante Thatsache, dafs sich in diesem Fall unter allen Umständen nur Chlortoluol bildet. Selbst wenn man die Flüssigkeit zum Kochen erhitzt, entsteht doch kein Chlorbenzyl. Die Reindarstellung des letzteren bietet nach dem Obigen keine Schwierigkeiten. - Sobald durch siedendes Toluol Chlor geleitet wird, entsteht nur Chlorbenzyl. Man braucht nicht einmal stets die Flüssigkeit im vollen Sieden zu erhalten. Wir haben gefunden, dafs schon eine verhältnifsmärsig geringe Erwärmung hiureicht, um die Bildung des Chlortoluols zu verhindern, wie denn überhaupt die Darstellung des Chlorbenzyls viel leichter und bequemer gelingt; als diejenige des Chlortoluols.

\section{Chlortaluol, $\mathrm{C}_{6} \mathrm{H}_{4} \mathrm{Cl}\left(\mathrm{CH}_{3}\right)$.}

Die Bildung und Darstellung dieses Körpers ist oben erläutert. Man leitete in mit Jod versetztes Toluol die berechnete Menge Chlor. Das Product wird der fractionirten Destillation unterworfen, das unter $140^{\circ}$ Uebergehende wieder mit Chlor behandelt und so fortgefahren, bis eine genügende Menge über $140^{\circ}$ siedender Substanz vorhanden ist. Man gewinnt dann leicht zwischen 157 und $158^{\circ}$ siedendes Chlortoluol, welches noch durch etwas Jod verunreinigt isi. Es ist ein Uebelstand bei dieser Methode, dafs sich neben den Chlorsubstitutionsproducten gleichzeitig Jodderivate bilden; die nur schwer zu entfernen sind. Am Besten wahrschemlich noch durch Erhitzen des Präparates mit Jodwasserstoff. Wir haben unser Chlortoluol, zur Entfernung der Jodbeimengung, so lange in die Sonne gestellt, bis dadurch kein Jod mehr in Freiheit gesetzt wurde. Dann wurde mit Kali gewaschen, entwässert und rectificirt.

1) 0,2047 Grm. gaben 0,4965 $\mathrm{CO}_{2}$ und $0,1019 \mathrm{H}_{8} \mathrm{O}$.

2) 0,2030 Grm. gaben 0,2278 $\mathrm{AgCl}$. 


\begin{tabular}{|c|c|c|c|c|}
\hline & \multicolumn{2}{|c|}{ Berechnet } & \multicolumn{2}{|c|}{ Gefunden } \\
\hline & & & 1) & 2) \\
\hline $\mathrm{C}_{7}$ & 84 & 66,4 & 66,2 & - \\
\hline $\mathbf{H}_{7}$ & 7 & 5,5 & 5,5 & - \\
\hline $\mathrm{Cl}$ & 35,5 & 28,1 & - & 27,8 \\
\hline & 126,5 & 100,0 & & \\
\hline
\end{tabular}

Das Chlortoluol zeigt gegen Reagentien dieselbe Beständigkeit wie das Chlorbenzol. Es wird weder von Cyankalium, noch Schwefelkalium oder Silbersalzen angegriffen. Löst man es in rauchender Salpetersäure, so erhält man NitroChlortoluol, das flüssig ist und sich destilliren läfst. Die interessanteste Umwandlung erleidet es durch Chromsäure, es wird hierdurch in Chlordracylsäure $\mathrm{C}_{6} \mathrm{H}_{4} \mathrm{Cl}(\mathrm{CO}$. HO) übergeführt, eine Reaction, die vollständig mit dem Verhalten des Nitrotoluols übereinstimmt.

Kocht man Toluol mit rauchender Salpetersäure, so bildet sich neben Nitrobenzö̈säure die mit dieser isomere Nitrodracylsäure. Die Entstehung beider Säuren erklärt sich daraus, dafs einerseits Toluol zu Benzoësäure oxydirt wird, welche dann in Nitrobenzoësäure übergeht. Andererseits geht aber die Hauptmenge des Toluols in Nitrotoluol über, welches offenbar durch die rauchende Salpetersäure in Nitrudracylsäure verwandelt wird. Letztere Säure erscheint daher als das einfache Oxydationsproduct des Nitrotoluols, und es war vorauszusehen, dafs sie auch auf andere Weise aus dem Nitrotoluol zu erhalten sein würde. Der Versuch hat dieses vollkommen bestätigt. Herr Dr. W. Dammann hat schon früher auf unsere Veranlassung Nitrotoluol mit Chromsäuremischung behandelt und dadurch sehr reine Nitrodracylsäure erhalten. Man erhitzt dazu in einem mit Kühlrohr versehenen Kolben ein Gemenge von 10 Thln. Nitrotoluol, 10 Thln. Kalium-Bichromat und 55 Theilen Schwefelsäure, die mit dem doppelten Volumen Wasser verdünnt wird. Das Nitro- 
toluol wird rasch angegriffen; sobald das Gemisch grün geworden ist, destillirt man das unangegriffene Nitrotoluol mit den Wasserdëmpfen ab, filtrirt den Rückstand nach dem Erkalten und kocht ihn wiederholt mit Sodalösung aus. Die alkalischen Lösungen werden concentrirt und mit Salzsäure gefältt. Die gefällte Säure war schon nach einmaligem Umkrystallisiren vollkommen rein. Sie zeigte den Schmelzpunkt $\left(238^{\circ}\right)$ und alle Eigenschaften der Nitrodracylsäure. Es wurde daraus das Kalksalz dargestellt und analysirt. Das bei $115^{\circ}$ getrocknete Salz enthielt $10,9 \mathrm{pC}$. Ca, berechnet 10,8 pC. Ca.

Die Oxydation des Nitrotoluols mit Chromsäure giebt ein sehr bequemes Mittel $a b$, urn sich rasch reine Nitrodracylsäure zu verschaffen. Mit grofsem Nutzen wendet man dasselbe Verfahren an, um die anderen Derivate und Homologen der Nitrodracylsäure darzustellen.

Behandelt man Chlortoluol in derselben Weise mit Chromsäurelösung, so wird es in Chlordracylsäure $\mathrm{C}_{7} \mathrm{H}_{5} \mathrm{ClO}_{2}$ übergeführt. Die durch wiederholtes Lösen in Ammoniak und Fällen der stark verdünnten Lösung mit Salzsäure gereinigte Säure zeigte den Schmelzpunkt $\left(236^{\circ}\right)$ und alle Eigenschaften der schon früher ${ }^{*}$ ) aus Azo-Amidodracylsäure erhaltenen Chlordracylsäure.

0,2404 Grm. gaben 0,2209 AgCl.

$$
\begin{array}{ccc} 
& \text { Berechnet } & \text { Gefunden } \\
\text { Cl } & 22,7 & 22,7 .
\end{array}
$$

Das daraus dargestellte chlordracylsaure Calcium zeigte die Krystallform, Löslichkeit und denselben Wassergehalt wie das früher erhaltene Salz $\left.{ }^{*}\right)\left(\mathrm{C}_{7} \mathrm{H}_{4} \mathrm{ClO}_{2}\right)_{2} \mathrm{Ca}+3 \mathrm{H}_{\mathbf{8}} \mathrm{O}$.

0,5191 Grm. gaben 0,0700 CaO.

$\begin{array}{ccc} & \text { Berechnet } & \text { Gefunden } \\ \text { Ca } & 9,9 & 9,6 .\end{array}$

*) Diese Annalen CXXXIII, 243. 
Es ergiebt sich also aus diesem Versuch, dafs im Nitrotoluol die Untersalpetersäure an derselben Stelle steht, wie im Chlortoluol das Chlor.

\section{Chlorbenzyl, $\mathrm{C}_{6} \mathrm{H}_{5}\left(\mathrm{CH}_{2} \mathrm{Cl}\right)$.}

Dieses ist der bisher Chlortoluol genannte Körper. Seine Darstellungsweise wurde oben angeführt. Derselbe unterscheidet sich vom isomeren Chlortoluol nicht blofs durch seinen höheren Siedepunkt, sondern auch durch die Leichtigkeit, mit welcher das Chlor aus demselben eliminirt werden kann. Er dient als Ausgangspunkt für die Darstellung der Benzylverbindungen. Nicht minder characteristisch ist sein Verhalten gegen Chromsäure. Es wird dadurch leicht und vollständig in Benzoësäure übergeführt. Verfährt man in der mehrfach angedeuteten Weise und reinigt die Säure in passender Art, so erhält man chemisch reine Benzoösäure. Wir haben zum Ueberflufs noch das Calciumsalz dargestellt und analysirt. Es zeigte sich völlig übereinstimmend mit benzoësaurem Calcium $\left(\mathrm{C}_{7} \mathrm{H}_{5} \mathrm{O}_{2}\right)_{2} \mathrm{Ca}+3 \mathrm{H}_{2} \mathrm{O}$.

$0,6656 \mathrm{Grm}$. verloren bei $120^{\circ} 0,1029 \mathrm{H}_{2} \mathrm{O}$ und gaben $0,1119 \mathrm{CaO}$.

\begin{tabular}{|c|c|c|c|}
\hline \multirow[b]{2}{*}{$\left(\mathrm{C}_{7} \mathrm{H}_{5} \mathrm{O}_{2}\right)_{2}$} & \multicolumn{2}{|c|}{ Berechnet } & \multirow{2}{*}{ Gefunden } \\
\hline & 242 & 85,8 & \\
\hline \multirow[t]{2}{*}{$\mathrm{Ca}$} & 40 & 14,2 & 14,2 \\
\hline & 282 & 100,0 & \\
\hline \multirow[t]{2}{*}{$3 \mathrm{H}_{2} \mathrm{O}$} & 54 & 16,1 & 15,5 \\
\hline & 33 & & \\
\hline
\end{tabular}

3. Nitro-Benzylchlorid, $\mathrm{C}_{6} \mathrm{H}_{4}\left(\mathrm{NO}_{z}\right)\left(\mathrm{CH}_{2} \mathrm{Cl}\right)$.

Löst man Chlorbenzyl in rauchender Salpetersäure, so fallt auf Zusatz von Wasser zu dieser Lösung ein Oel heraus, das bald zu einer weichen Masse erstarrt. Bringt man dieselbe auf ein trockenes Filter, so bleiben Krystalle zurück, wåhrend zugleich eine nicht unbedeutende Menge eines Oeles abtropft. Die Krystalle werden zwischen Fliefspapier abgeAnnal. d. Chem. u. Pharm. CXXXIX. Bd. 3. Heft. 
prefst und wiederholt aus Alkohol umkrystallisirt. Man erhält sehr schöne glänzende blätterige Krystalle, die bei $71^{0}$ schmelzen. Sie sind nitrirtes Benzylchlorid.

1) $0,2295 \mathrm{Grm}$. gaben $0,4178 \mathrm{CO}_{2}$ und $0,0865 \mathrm{H}_{2} \mathrm{O}$.

2) 0,2660 Grm. gaben $0,215 \mathrm{AgCl}$.

\begin{tabular}{lcrrrr}
\multicolumn{2}{c}{ Berechnet } & & \multicolumn{2}{c}{ Gefunden } \\
\hline $\mathrm{C}_{7}$ & 84 & 49,0 & 1 ) & 2 ) \\
$\mathrm{H}_{6}$ & 6 & 3,5 & 49,6 & - \\
$\mathrm{Cl}$ & 35,5 & 20,7 & 4,2 & - \\
$\mathrm{N}$ & 14 & 8,1 & - & 20,0 \\
$\mathrm{O}_{2}$ & 32 & 18,7 & - & - \\
\cline { 2 - 5 } & 171,5 & $100,0$. & & & -
\end{tabular}

Da Chlorbenzyl leicht in Benzylalkohol und in Benzoësäure übergeführt werden kann, so glaubten wir anfangs, das nitrirte Chlorbenzyl gehòre der Reihe der Nitrobenzö̈säure an. Behandelt man es aber mit Chromsäure, so geht es sehr leicht in Nitrodracylsäure über. Demnach übt das Chlor im Methyl des Toluols keinen Einflufs auf die Stellung der Untersalpetersëure im Phenyl aus.

Es wurde oben erwähnt, dafs neben den Krystallen des nitrirten Chlorbenzyls stets viel eines Oeles gewonnen wird. Nach langem Stehen scheiden sich aus demselben noch Krystalle von Nitro-Benzylchlorid aus. Da es in Alkohol viel weniger löslich ist, als letzteres, so wurde es durch wiederholtes Lösen in heifsem Alkohol und Ausscheiden beim Erkalten zu reinigen gesucht. Bei der Destillation zersetzt es sich. Eine vorläufige Analyse ergab $51,8 \mathrm{pC}$. C und 4,0 pC. H. Ueber die Natur desselben werden wir später berichten und wollen einstweilen nur mittheilen, dafs es beim Behandeln mit Chromsäure ein Säuregemisch liefert, von welchem der gröfste Theil ebenfalls aus Nitrodracylsäure bestand.

Man kann das Nitro-Benzylchlorid betrachten als die Chlorverbindung eines Nitrobenzylalkohols : 


$$
\mathrm{C}_{6} \mathrm{H}_{4}\left(\mathrm{NO}_{2}\right)\left(\mathrm{CH}_{2} \cdot \mathrm{HO}\right) \text {. }
$$

Wir würden darin den Repräsentanten eines nitrirten Alkohols besitzen, wie das Nitro-Bittermandelöl der zur Nitrobenzoēsãure gehörige Aldehyd ist. Vom Nitro-Alkohol ausgehend werden sich dann mehrere Reihen neuer organischer Verbindungen darstellen lassen. Wir sind gegenwärtig mit der Untersuchung aller dieser Körper beschäftigt.

Seitdem das Toluol als Methylbenzol $\mathrm{C}_{6} \mathrm{H}_{5}\left(\mathrm{CH}_{3}\right)$ erkannt worden ist, bietet die Erklärung der im Obigen aufgezählten Thatsachen keine Schwierigkeiten mehr. Behandelt man das Toluol in der Kälte, oder bei Gegenwart von Jod, mit Chlor, so tritt das Chlor an die Stelle von Wasserstoff im Phenyl, und man erhält Chlortoluol $\mathrm{C}_{6} \mathrm{H}_{4} \mathrm{Cl}\left(\mathrm{CH}_{3}\right)$, in welchem das Chlor also dieselbe Stellung hat wie im gechlorten Benzol. Der Körper hält daher das Chlor mit derselben Festigkeit gebunden wie das gechlorte Benzol. Das Verhalten des Chlortoluols gegen Chromsäure ergiebt sich aus der Gleichung :

$$
\begin{aligned}
& \mathrm{C}_{6} \mathrm{H}_{4} \mathrm{Cl}\left(\mathrm{CH}_{8}\right)+\mathrm{O}_{8}=\mathrm{C}_{8} \mathrm{H}_{4} \mathrm{Cl}(\mathrm{CO} . \mathrm{HO})+\mathrm{H}_{8} \mathrm{O} \\
& \text { Chlortoluol Chlordracylsäure. }
\end{aligned}
$$

Behandelt man das Toluol in der Siedehitze mit Chlor, so tritt das Chlor an die Stelle von Wasserstoff im Methyl, man erhält Chlorbenzyl $\mathrm{C}_{6} \mathrm{H}_{5}\left(\mathrm{CH}_{2} \mathrm{Cl}\right)$, in welchem also das Chlor dieselbe Stellung einnimmt, wie im Chlormethyl und überhaupt in den s. g. Fettkörpern. Das Chlor ist daher auch eben so locker gebunden wie in diesen, und kann leicht in doppelten Zersetzungen ausgewechselt werden. Die 0xydation des Chlorbenzyls durch Chromsäure wird durch folgende Gleichungen veranschaulicht :

$$
\begin{aligned}
& \mathrm{C}_{6} \mathrm{H}_{5}\left(\mathrm{CH}_{2} \mathrm{Cl}\right)+\mathrm{O}_{2}=\mathrm{C}_{6} \mathrm{H}_{5}(\mathrm{CO} . \mathrm{Cl})+\mathrm{H}_{2} \mathrm{O} \text { und } \\
& \text { Chlorbenzyl Chlorbenzoyl } \\
& \mathrm{C}_{8} \mathrm{H}_{5}(\mathrm{CO} . \mathrm{Cl})+\mathrm{H}_{2} \mathrm{O}=\mathrm{C}_{6} \mathrm{H}_{5}(\mathrm{CO} \cdot \mathrm{HO})+\mathrm{HCl} \text {. } \\
& \text { Benzoësäure. }
\end{aligned}
$$

Brom scheint auf die Homologen des Benzols viel einfacher einzuwirken, ajs Chlor. Aus den Eigenschaften des 
von Glinzer und Fittig beschriebenen *) Mono-Bromtoluols ergiebt sich, daf's diesem Körper die rationelle Formel $\mathrm{C}_{6} \mathrm{H}_{4} \mathrm{Br}\left(\mathrm{CH}_{3}\right)$ zukommt. Ein dem Chlorbenzyl entsprechendes Brombenzyl $\mathrm{C}_{6} \mathrm{H}_{5}\left(\mathrm{CH}_{2} \mathrm{Br}\right)$ wurde aus theoretischen Gründen vermuthet. Es ist inzwischen von Kekulé**) aus Benzylalkohol dargestellt worden.

$\begin{array}{llllll}\text { Chlortoluol } & \mathrm{C}_{6} \mathrm{H}_{4} \mathrm{Cl}\left(\mathrm{CH}_{3}\right) & 157^{\circ} & \text { Chlorbenzyl } & \mathrm{C}_{6} \mathrm{H}_{5}\left(\mathrm{CH}_{2} \mathrm{Cl}\right) & 176^{\circ} . \\ \text { Bromtoluol } & \mathrm{C}_{6} \mathrm{H}_{4} \mathrm{Br}\left(\mathrm{CH}_{3}\right) & 179^{\circ} & \text { Brombenzyl } & \mathrm{C}_{8} \mathrm{H}_{6}\left(\mathrm{CH}_{2} \mathrm{Br}\right) & 199^{\circ} .\end{array}$

Es erklärt sich nun sehr natürlich, warum das Radical Benzyl, $\mathrm{C}_{6} \mathrm{H}_{5}\left(\mathrm{CH}_{2}\right)$, nur aus s. g. Chlortoluol (Chlorbenzyl), nicht aber aus Bromtoluol dargestellt werden konnte. Eben so warum wiederum umgekehrt zur Darstellung von Methyloder Aethyl-Toluol nur Bromtoluol, nicht aber Chlorbenzyl tauglich ist ***). Man hat in der That : $\mathrm{C}_{6} \mathrm{H}_{4} \mathrm{Br}\left(\mathrm{CH}_{3}\right)+$ $\mathrm{CH}_{3} \mathrm{~J}+\mathrm{Na}_{2}=\mathrm{C}_{6} \mathrm{H}_{4}\left(\mathrm{CH}_{3}\right)_{2}+\mathrm{NaJ}+\mathrm{NaBr}$.

\section{Dichlortoluol, $\mathrm{C}_{6} \mathrm{H}_{3} \mathrm{Cl}_{2}\left(\mathrm{CH}_{3}\right)$.}

Ueber Dichlortoluol liegen bereits einige Untersuchungen vor $f)$. Nach den neuen Erfahrungen ist aber der Werth dieser Arbeiten ein sehr untergeordneter, da man offenbar stets mit einem Gemenge verschiedener isomerer Derivate gearbeitet hat. Berücksichtigt man die so sehr verschiedenen Eigenschaften der Substitutionsproducte des Toluols, welche sich aus der verschiedenen Stellung des Chlors im Toluol ergeben, so sind folgende drei isomere Fälle für Dichlortoluol möglich :

$\begin{array}{ccc}\mathrm{C}_{6} \mathrm{H}_{3} \mathrm{Cl}_{2}\left(\mathrm{CH}_{3}\right) & \mathrm{C}_{6} \mathrm{H}_{4} \mathrm{Cl}\left(\mathrm{CH}_{2} \mathrm{Cl}\right) & \mathrm{C}_{6} \mathrm{H}_{5}\left(\mathrm{CHCl}_{4}\right) \\ \text { Dichlor-Toluol } & \text { Gechlortes Cblorbenzyl } & \text { Benzylai-Chlorid. }\end{array}$

*) Diese Annalen CXXXVI, 301.

**) Daselbst CXXXVII, 190.

***) Daselbst CXXXVI, 303.

†) Daselbst CXVI, 336 und Supplementbd. II. 
Von diesen drei Isomeren ist das Benzylal-Chlorid *) (Bittermandelöl-Chlorid) am Genauesten untersucht. Seine beiden Chloratome treten bei doppelten Zersetzungen mit grofser Leichtigkeit aus. - Vielleicht entsteht es, wenn man Chlor anhaltend in siedendes Toluol leitet.

Was bisher Dichlor-Toluol genannt wurde, scheint vorzugsweise aus gechlortem Benzylchlorid bestanden zu haben. So erhielt der Eine von uns früher **), beim Behandeln dieses Körpers mit Schwefelwasserstoff-Schwefelkalium, schöne glänzende Krystalle eines Körpers $\mathrm{C}_{7} \mathrm{H}_{7} \mathrm{ClS}$.

$$
\mathrm{C}_{8} \mathrm{H}_{4} \mathrm{Cl}\left(\mathrm{CH}_{2} \mathrm{Cl}\right)+\mathrm{KHS}=\mathrm{C}_{6} \mathrm{H}_{4} \mathrm{Cl}\left(\mathrm{CH}_{2} . \mathrm{HS}\right)+\mathrm{KCl} \text {. }
$$

Und Naquet***) stellte durch Behandeln des s. g. Dichlortoluols mit alkoholischem Kali einen Körper $\mathrm{C}_{9} \mathrm{H}_{11} \mathrm{ClO}$ dar.

$$
\mathrm{C}_{6} \mathrm{H}_{4} \mathrm{Cl}\left(\mathrm{CH}_{2} \mathrm{Cl}\right)+\mathrm{C}_{2} \mathrm{H}_{5} \mathrm{KO}=\mathrm{C}_{6} \mathrm{H}_{4} \mathrm{Cl}\left(\mathrm{CH}_{2} \cdot \mathrm{C}_{2} \mathrm{H}_{5} \mathrm{O}\right)+\mathrm{KCl} \text {. }
$$

Ein reines gechlortes Chlorbenzyl wird sich darstellen lassen entweder durch Bebandeln von Chlortoluol mit Chlor in der Siedehitze, oder durch Einleiten von Chlor in mit Jod versetztes Chlorbenzyl. Vielleicht sind aber die auf diese zwei Arten erhaltenen Producte nur isomer. Ein Körper $\mathrm{C}_{6} \mathrm{H}_{4} \mathrm{Cl}\left(\mathrm{CH}_{2} \mathrm{Cl}\right)$ könnte z. B. der Reihe der Chlorbenzoësäure, der andere derjenigen der Chlordracylsäure entsprechen.

Dichlortoluol $\mathrm{C}_{6} \mathrm{H}_{3} \mathrm{Cl}_{2}\left(\mathrm{CH}_{3}\right)$ entsteht leicht, wenn Chlor bei Gegenwart von Jod auf Toluol einwirkt. Man erhält eine bei etwa $196^{\circ}$ siedende Flüssigkeit, in welcher beide Cliloratome mit derselben Festigkeit gebunden sind, wie im Chlor-

*) Der Name Chlorbenzol für diesen Körper kann, ohne zu steten Verwechselungen Veranlassung zu geben, nicht länger beibebalten werden.

**) Diese Annalen CXVI, 346.

***) Daselbst Supplementbd. II, 250. 
342 Beilstein u. Geitner, über das Verhalten u. s. w.

toluol. Behandelt man Dichlortoluol mit Chromsäure, so erhält man Dichlordracylsäure, $\mathrm{C}_{7} \mathrm{H}_{4} \mathrm{Cl}_{2} \mathrm{O}_{2}$, nach der Gleichung :

$$
\underset{\text { Dichlortoluol }}{\mathrm{C}_{8} \mathrm{H}_{3} \mathrm{Cl}_{2}\left(\mathrm{CH}_{3}\right)+\mathrm{O}_{3}=\underset{\text { Dichlordracylshure. }}{\mathrm{C}_{6} \mathrm{H}_{3} \mathrm{Cl}_{8}(\mathrm{CO} . \mathrm{HO})}+\mathrm{H}_{2} \mathrm{O}}
$$

Die ausführliche Beschreibung der Isomeren $\mathrm{C}_{7} \mathrm{H}_{6} \mathrm{Cl}_{2}$ und der höheren Chlorderivate des Toluols wird den Gegenstand einer nächsten Abhandlung bilden.

Was wir vom Toluol nachgewiesen haben, bleibt nicht auf diesen Kohlenwasserstoff beschränkt. Herr Vollrath hat bereits aus dem Xylol zwei Derivate $\mathrm{C}_{6} \mathrm{H}_{4} \int_{\mathrm{CH}_{2} \mathrm{Cl}}^{\mathrm{CH}_{3}}$ und $\mathrm{C}_{6} \mathrm{H}_{3} \mathrm{Cl} / \mathrm{CH}_{3} \mathrm{CH}_{3}$ dargestellt, welche genau in derselben Beziehung zu einander stehen, wie Chlortoluol und Chlorbenzyl. Für das Cumol findet zweifelsohne dasselbe statt. Auch wäre es interessant, die Homologen des Sumpfgases in gleicher Weise zu untersuchen. Wenden wir unsere Erfahrungen auf andere Kohlenwasserstoffe an, z. B. Naphtalin, so werden sich nun die zahlreichen isomeren Chlornaphtaline Laurent's in einfacher Weise einzeln rein darstellen lassen. Das abwechselnde Behandeln der Kohlenwasserstofe mit Chlor in der Siedehitze und in der Kälte, oder bei Gegenwart von Jod, giebt daher einen neuen und erfolgreichen Weg an zur Erforschung der Constitution der aromatischen Verbindungen.

Laboratorium in Göttingen. 